\title{
A Shipping Noise Prediction Tool
}

\author{
Cristiano Soares ${ }^{a}$, Friedrich Zabel $^{a)}$, Sérgio M. Jesus ${ }^{b)}$ \\ ${ }^{a)}$ MarSensing Lda., Centro Empresarial Pav. A5, Campus de Gambelas, 8005-139 Faro, Portugal \\ ${ }^{b)}$ LARSyS, University of Algarve, Campus de Gambelas, 8005-139 Faro, Portugal \\ e-mail: csoares@marsensing.com
}

\begin{abstract}
Underwater noise is a form of pollution causing significant concern in terms of environmental status. Shipping is considered the main contributor to the total noise at the global scale, since ship radiated noise can propagate up to tens or hundreds of kilometers. This paper reports on a shipping noise prediction tool based on Automatic Identification System (AIS) data and a normal-mode acoustic propagation code. The tool is set up with realistic environmental information and continuously fed with AIS data, as to periodically produce an instantaneous picture of noise level due to point noise sources present in the area under observation. This produces a time series, that is used for statistical post-processing in order to obtain statistical indicators for a meaningful noise assessment. As a case study, experimental results are reported for a regional observation area in Portuguese waters, off the Southwest Coast of Portugal, crossed by heavy shipping routes. Shipping noise maps are generated for AIS data collected over a 9 month interval. Although the results are yet to be validated by terrain acoustic measurements, this exercise illustrates that a great deal of meaningful information on noise distribution can be obtained by using appropriate acoustic propagation models coupled with live AIS data into a handy noise prediction tool, made available at http://www.shippingnoise.com.
\end{abstract}

\section{INTRODUCTION}

Underwater noise is a form of pollution that has been an increasing source of concern among the scientific community and environmental protection organisations in terms of environmental status, in the last two decades. Among different sources of underwater noise, shipping is considered the main contributor to the total noise at global scale. An estimated number of about 100 thousand ships simultaneously cruise the seas around the world. The total gross tonnage of ships quadrupled between 1965 and 2003, in the same time the number of commercial ships approximately doubled [1]. Shipping noise is generated in different ways during ship operation, most notably from propeller cavitation, with most spectral contents below $1 \mathrm{kHz}$. Since ship radiated noise can propagate up to tens or hundreds of kilometers, it is acceptable to hypothesize that one of the most relevant environmental effects is masking of biologically relevant sounds, such as sounds vocalised by marine animals for mating, or for detection of prays or predators.

Several studies on shipping noise were carried out as an attempt to characterize the soundscape in sensitive marine environments [2], or for noise source characterization [1], [3]. In Reference [2], the authors characterize the contribution of large vessels to the total noise in the Stellwagen Bank National Marine Sanctuary, and claimed that noise produced by large commercial vessels attained levels that could prevent whales to maintain acoustic contact within the sanctuary. McKenna et al. [1] conducted measurements of ship radiated noise in the Santa Barbara Channel (southern California) by opportunity recordings collected with a moored acoustic recorder and Automatic Identification System (AIS) data. Wales and Heitmeyer [3] presented an evaluation of the classical model for determining an ensemble of the broadband source spectra of the sound generated by individual ships and proposed an alternate model to overcome the deficiencies in the classical model, which could signficantly reduce the rms error to an ensemble of 54 measured source spectra.

Direct measurement of shipping noise is a very challenging task for the difficulty in equipment standardization, and for the coverage requirement of large extensions of international waters and countries EEZ as, for example, the Portuguese EEZ of about 1.7 million $\mathrm{km}^{2}$. Recently, shipping noise modelling is being proposed to overcome these challenges. After the European Union has approved the Marine Strategy Framework Directive (MSFD) [4], the Technical Subgroup on Underwater Noise and Other Forms of Energy proposed indicators for underwater noise and recommended the application of acoustic propagation modelling tools as a strategy to overcome spatial predictions that are not practicable with in situ measurements only [5]. Meanwhile, some authors have implemented prediction tools for ship noise. Porter et al. [6] have implemented a tool for the prediction of the global soundscape based on Source Level density, which is converted into equispaced virtual sources to be convolved with channel acoustic responses. Colin et al. [7] have proposed a noise prediction tool based on Automatic Identification System (AIS) and biological distribution data in order to generate representative sound exposure maps.

This paper reports on a shipping noise prediction tool based on AIS data and a normal-modes acoustic propagation model. The tool is set up with realistic environmental information and continuously fed with AIS data, as to periodically produce an instantaneous picture of noise level due to point noise sources present in the area under observation. This produces a time series, which is used for post-processing in order to obtain statistical indicators such as average noise levels and noise level distributions.

The actual noise prediction tool has been computing instantaneous noise maps since February 2012, for the Portuguese waters off the Southwest coast of Portugal, in an area crossed by heavy shipping routes. Since then, noise and postprocessing pictures were continuously delivered to an online 
site at http://www.shippingnoise.com. This area is adopted herein as a case study, where experimental results for 9 month of AIS data are reported.

\section{The Shipping Noise Prediction Tool}

This section presents a shipping noise prediction tool based on AIS data, and describes three main aspects: the AIS data input; the source level input; and the acoustic model used for field computations.

\section{A. The AIS data input}

Shipping information is retrieved from AIS data collected with our own AIS receiver and from an AIS data sharing service (MarineTraffic.com). The overall AIS data is searched for the ships cruising within the geographical boundaries of interest. The processed AIS tag provides several relevant parameters for each ship contained in the selected ocean area, including current position, ground speed, ship type, draught, status, etc.

Assuming propeller cavitation as the main source of noise produced by a cruising ship, the depth at which the propeller is located remains one of the crucial parameters to be inserted in the sound propagation model. In practice, propeller depth is unknown, since it depends on the ships' load and draught. Altough draught is a parameter included in the AIS data format, in general, it is not expected that this parameter is up to date. Another difficulty may arise from the fact that besides propeller cavitation, a number of other noise generating mechanisms may contribute significantly to the total ship radiated noise. In the literature, authors refer to a depth interval from 7 to $14 \mathrm{~m}$ for model input. The AIS data is organized in time slots with a selected length, and treated such that each identified ship appears only once within each time slot.

\section{B. Source spectra}

To compute the noise propagated by each ship, a description of Source Level (SL) is required, which is to be convolved with the acoustic response of the propagation media between the source and a given measurement position. In the actual study, an ensemble of shipping noise spectra was considered for acoustic modelling. These noise spectra, taken from Reference [1], are presented as octave-band levels with centre frequencies of 32, 63, 126, 252, 504, and $1008 \mathrm{~Hz}$. The AIS data does not discriminate ships as presented in Fig. 1, providing only a broader classification, such as cargo, tanker or passenger, etc. The spectrum selection algorithm first selects ship type, and then selects a spectrum based on AIS ground speed.

\section{Field computations}

The noise prediction tool combines SL with transmission loss (TL), in order to yield SPL due to an individual ship at a given position. TL calculations are carried out with the KRAKEN normal mode computer model [8], [9]. A normalmode model is computationally very convenient for the actual
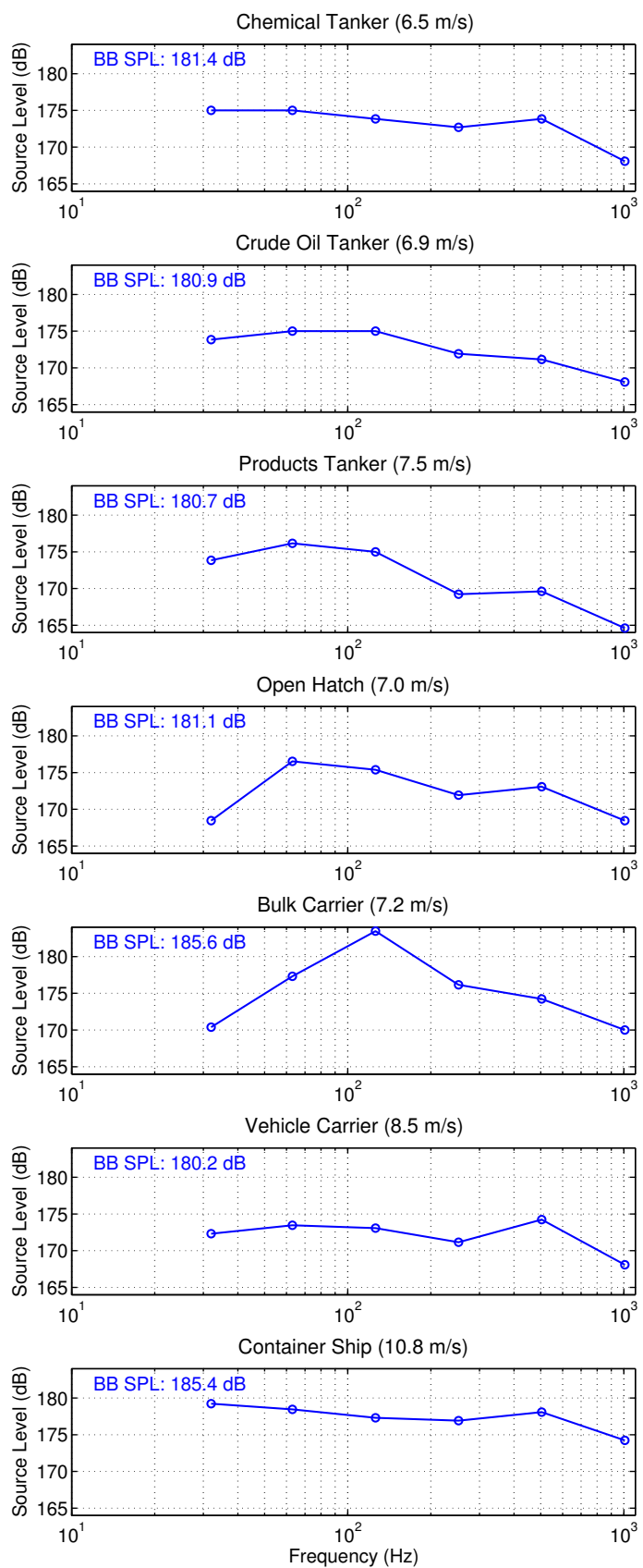

Fig. 1. One-octave band source levels reproduced from Ref. [1] for: three types of tankers (top panels), open hatch cargos and bulk carriers (middle panels), and vehicle carriers and container ships (bottom panels).

concept, since the normal modes need to be computed only when a new set of environmental parameters are defined. Ships are modelled as point sources, and the KRAKEN model is set up to output incoherent TL over a given set of discrete frequencies, for positions 360 degrees around each source position within a given radius, and a depth, with given steps for angle and range, in order to yield a TL disc for each discrete frequency. For each frequency, the SL is combined with TL to produce the spectral power at that frequency. A final SPL disc is obtained by integration of the spectral density power 
as

$$
Y_{n}\left(R_{r}, \theta_{r}\right)=\sqrt{\sum_{k=1}^{K}\left|S\left(\omega_{k}\right)\right|^{2}\left|H_{n}\left(\omega_{k}, R_{r}, \theta_{r}\right)\right|^{2}},
$$

where $Y_{n}\left(R_{r}, \theta_{r}\right)$ is the rms SPL at a receiver at range $R_{r}$ and bearing $\theta_{r}$ due to the $n^{\text {th }}$ ship, $S\left(\omega_{k}\right)$ is the SL for a band with center frequency $\omega_{k}$ in $\mu \mathrm{Pa}$, and $H_{n}\left(\omega_{k}, R_{r}, \theta_{r}\right)$ is the TL between the $n^{\text {th }}$ ship and the position denoted by $R_{r}$ and $\theta_{r}$. The subscript ${ }_{r}$ stands for receiver. The number of frequencies $K$ will depend, in general, on the available bandwith, and the resolution considered (e.g. octave bands or $1 / 3$ octave bands, etc.). Finally, a snapshot of the sound map as a superposition of the radiated sound levels is obtained for all receiver positions over a predetermined spatial grid. For each noise source, the ships contained in the AIS data, a SPL disc for depth $80 \mathrm{~m}$ was computed. The resolution in range was $0.5 \mathrm{~km}$ (201 ranges), and the resolution in bearing was 6 degrees (61 bearings). Source depth was set to $9 \mathrm{~m}$ for all ships.

The prediction tool is fed with an environmental description, including bathymetry, a sound-speed profile, and the parameters of a bottom composed of a sediment layer over an infinite half-space with parameters taken from a table of sediment materials (wave attenuations, densities, and sound velocities).

The bathymetry of the study area is shown in Figure 2. The Continental Shelf is relatively steep exceeding water depth of $4000 \mathrm{~m}$ within 60 nautical miles from the West Coast of Portugal, and the maximum depth exceeds $5000 \mathrm{~m}$. The bathymetric data defines the working box, which covers a distance of approximately $315 \mathrm{~km}$ from West to East, and $338 \mathrm{~km}$ from South to North. The useful bathymetric area, relevant for field calculations, covers approximately $60500 \mathrm{~km}^{2}$.

The watercolumn is parameterised with a sound-speed profile based on a single CTD measurement taken in July 11,2007 , at latitude $38^{\circ} 10.350 \mathrm{~N}$ and longitude $09^{\circ} 19.158 \mathrm{~W}$, which was the deepest position of a series of measurement taken in July 2007 (Figure 3), during the RADAR'07 sea trial, in the area of the Setubal Canyon. The maximum depth of the sound speed profile is $1324 \mathrm{~m}$. For greater depths, temperature and salinity were assumed constant from the last CTD depth to the maximum water depth at each location in the study area. Often, the environmental input may be a major difficulty in long-term and large-scale acoustic prediction, due to environmental variablity. Heitmeyer et al. have shown that discrepancies in acoustic modelling may result from using historical environmental data, and model approximations that are not acceptable under some environmental scenarios [10]. This is a difficulty that arises in long-term and large-scale noise predictions, as a great deal of temporal and spatial input information is required.

The seafloor consisted of parameters representing a sandy sediment layer over a rocky infinite sub-bottom. Table I shows the seafloor parameters used for forward computations. As no knowledge on these parameters were available, the choice was based on values found in tables of geoacoustic properties [11].

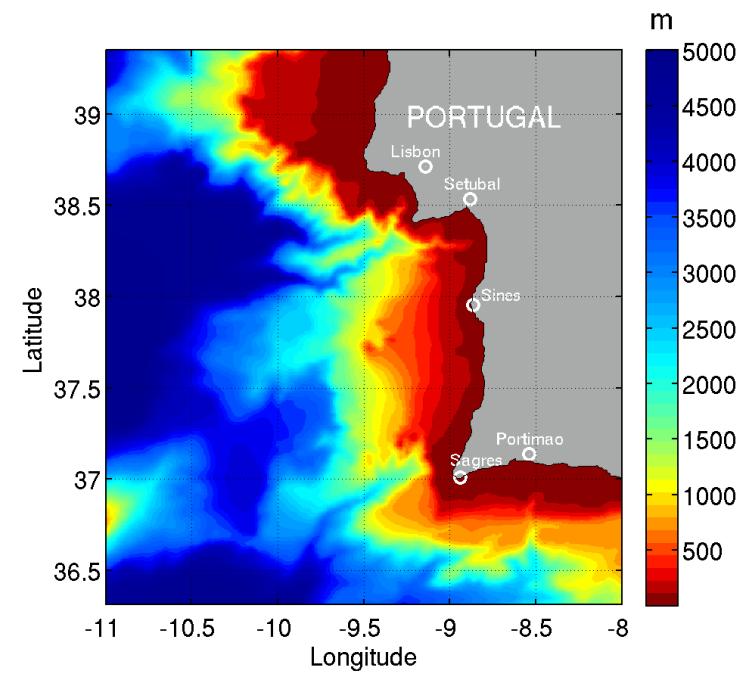

Fig. 2. Acoustic model environmental input data: bathymetry.

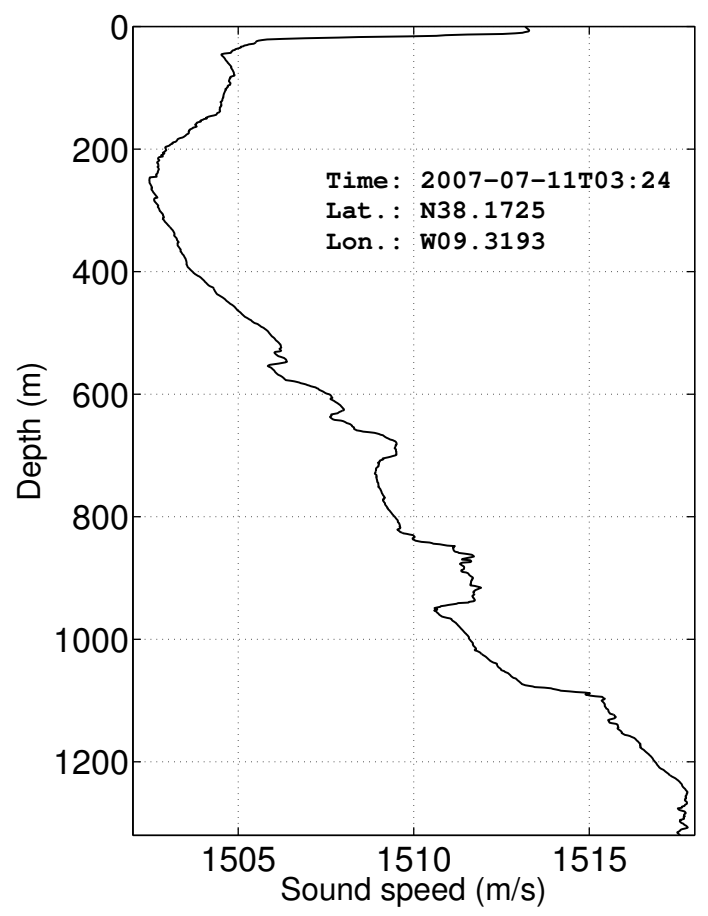

Fig. 3. Acoustic model environmental input data: sound speed profile.

The poor knowledge on the seafloor properties should have little influence on the accuracy of the acoustic modelling in most of the area, as only a relatively reduced fraction of the area is shallow water.

\section{EXPERIMENTAL RESULTS: SHIPPING NOISE MAPPING}

This section reports on experimental results on shipping noise mapping for an observation area in Portuguese waters off the Southwest Coast of Portugal, crossed by heavy shipping routes connecting the Mediterranean and South Atlantic to Northern Europe. 
TABLE I

PARAMETER VALUES FOR SEDIMENT LAYER AND SUB-BOTTOM LAYERS USED FOR FORWARD MODELLING OF TRANSMISSION LOSS.

\begin{tabular}{|l|c|}
\hline Model Parameter & \\
\hline Sediment speed $(\mathrm{m} / \mathrm{s})$ & 1650 \\
Sediment density $\left(\mathrm{g} / \mathrm{cm}^{3}\right)$ & 1.9 \\
Sediment att. $(\mathrm{dB} / \lambda)$ & 0.8 \\
Sediment thickness $(\mathrm{m})$ & 4 \\
\hline Sub-bottom speed $(\mathrm{m} / \mathrm{s})$ & 1800 \\
Sub-bottom density $\left(\mathrm{g} / \mathrm{cm}^{3}\right)$ & 2.0 \\
Sub-bottom att. $(\mathrm{dB} / \lambda)$ & 0.6 \\
\hline
\end{tabular}

\section{A. The AIS data set}

The AIS data used for shipping noise modelling covers an interval from March $19^{\text {th }}$ to December $30^{\text {th }}, 2014$ (over 9 month), in a deep water area, favorable to the propagation of low frequency acoustic waves. Figure 4 shows the cumulated shipping density over the longitude/latitude plane in $\log _{10}$ (ship $\times$ hour), in order to illustrate the importance of this area in terms of shipping routes, and for later reference. To compute shipping density, the area was gridded into cells with a resolution of 1 minute in longitude and latitude, and the total time spent by ships in each cell was cummulated. The AIS data was segmented into time frames of 10 minutes, in order to generate AIS data samples with that step. The plot clearly

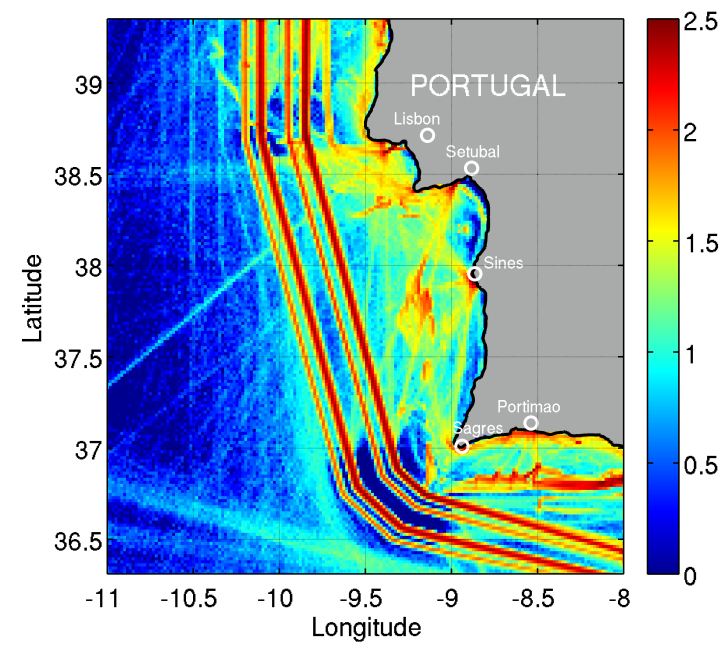

Fig. 4. Automatic Identification System data collected from March 19th to December 30th, 2014 (over 9 month): cumulated shipping density in $\log _{10}($ ship $\times$ hour $)$.

shows the main shipping lanes, where the maximum density was found, with more than 316 hours $\left(10^{2.5}\right.$ hours) spent in the corresponding cells. There are several other spots with intense shipping, which are related to the ports of Lisbon, Setúbal, and Sines. Off the South Coast, away from main shipping lanes, intense shipping is observed, which is mainly due to fishing ships, as well as some access lanes to the city of Portimão.

For the purpose of noise modelling, a total of about 38650 AIS data samples over the whole period were made available. AIS data segments with ship count below percentile 5 of the complete time series were excluded in order to cope with periods of poor AIS receiver coverage.

\section{B. Noise mapping}

The acoustic response $H_{n}$ in eq. (1) is calculated using the KRAKEN normal-modes acoustic propagation code [9]. For each ship, the inputs to the model are the emitter/receiver geometry and the environmental description given above, while the output is the frequency TL discs. The individual contribution to the noise map is computed with eq. (1) to yield a SPL disc, which is accumulated on a matrix containing the noise map. This procedure, repeated for each AIS data sample, which is regarded as a sampling process of the noise level over the longitude/latitude plane for the given depth, produces a time-series of instantaneous noise pictures. The choice for a 10 minutes sampling period is to enable sufficient sampling of the acoustic observable, while maintaining an affordable computation load and volume of synthetic data. The timeseries of instantaneous noise levels is subject to an a posteriori statistical processing, in order to obtain meaningful indicators such as average noise levels and noise level exceedance, for noise level assessment.

Figure 5 shows the average broadband sound pressure level (BB SPL) obtained over the area for a frequency band 32 to $1008 \mathrm{~Hz}$ and at a depth of $80 \mathrm{~m}$. The average sound pressure shows a significant outstanding level along the main shipping lanes, exceeding $120 \mathrm{~dB}$. Altough there is a clear relation between average SPL and shipping density, it is apparent that the noise propagation is mostly governed by the bathymetry, as the average noise level shows an assymetry with respect to the shipping lanes. On the Western side of the shipping lanes, the average noise level falls of less rapidly than on the Eastern side, where it is significantly attenuated for water depths below $100 \mathrm{~m}$. Localized spots of increased level are observed on the Eastern side of the shipping lanes, which

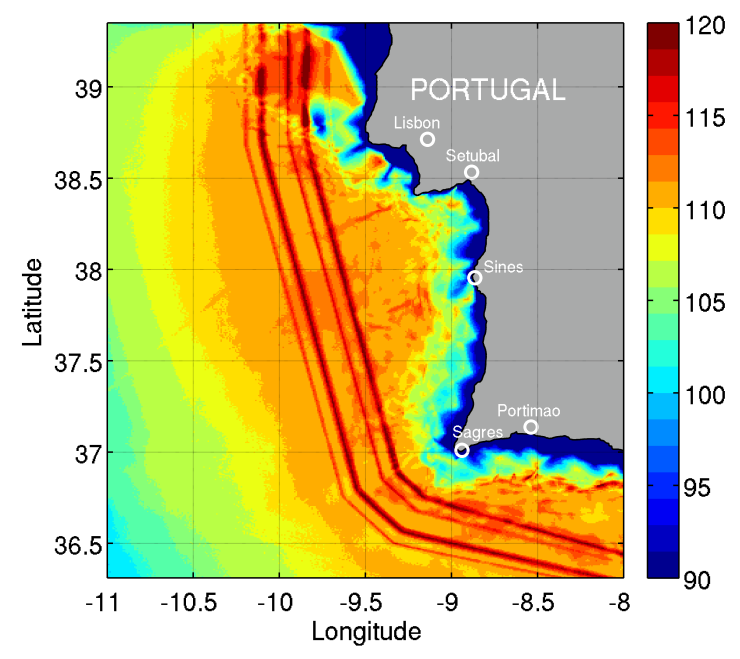

Fig. 5. Predicted average SPL [dB re to $1 \mu \mathrm{Pa}$ ] for at $80 \mathrm{~m}$ depth for a frequency band 32 to $1008 \mathrm{~Hz}$. The AIS data interval is from March 19 to December 30, 2014. 
is attributed to port access and local vessel activity. Similar effects are observed in the South Coast, where local fishing is relatively intense. Altough the actual problem is a large-scale issue, and the offshore strip with water depth below $100 \mathrm{~m}$ is only a fraction of the total area, it is important to take it into account for its importance in terms of biological production and diversity.

Another example, on how bathymetry significantly affects the noise propagation is in the area Northeast of Lisbon, where a spot of increased spreading of average noise level is observed, without significant increase of ship density. Here, the noise is being generated in shallow water, in comparison to the area Southeast of Lisbon. One must account for the significant range of wavelengths composing the signal model, as the frequency band extends from 32 to $1008 \mathrm{~Hz}$, and different environmental conditions may influence differently the propagation in the lower end and the upper end of the band. Shipping noise appears to be of non-stationary nature, due to the geometric dynamics of the noise sources (the ships) which can cause the sound exposure level of the marine environment to significantly change over time and space. Sound pressure averaging shall be complemented with statistical indicators that provide information on the exceeded levels. Level exceedance is meaningful in terms of percentage of time, as it is equivalent to determining a given percentile of all available SPL measurements. For example, percentile 5 of an SPL time-series is the value that is exceeded by $95 \%$ of the observations. Figure 6 shows noise level exceedance as a function of percentage of time. The level exceedance for 95\% of the time (top) and 5\% of the time (bottom), are useful for establishing lower and upper boundaries on a significant percentage of the total time, while level exceedance for $50 \%$ of the time (middle) shall be an indicator yielding values close to average, with the advantage of reducing the importance of outliers. The effect of the bathymetry on the propagation of noise is evident, especially in the level exceedance for $95 \%$ of the time, as the SPL over surface shows a red spot corresponding to 109 and $110 \mathrm{~dB}$ with a shape that can be identified in the bathymetric maps as the area where water depth is limited to $3000 \mathrm{~m}$, both off the West and South Coasts. This effect has a reduced outstanding for the other percentages of time. The level exceedance for $50 \%$ of the time (middle) is comparable to average SPL, however, with exclusion of the effect of the shipping lanes, as the outliers have a reduced contribution for this indicator, and with an offset of about $1 \mathrm{~dB}$. The exceedance level for $5 \%$ of the time (bottom) reveals peak levels observed around latitude $39^{\circ} 00.00 \mathrm{~N}$, an effect that is caused by intense shipping over the spot with reduced water depth. With this indicator a slight effect of the two heaviest shipping lanes can be observed, revealing that there is a peak SPL of short duration as ships cruise through.

Level exceedance can be presented for selected positions as the proportion of time where a given minimum level is reached or exceeded. This is presented in the form of a cummulative distribution, where SPL is plotted against percentage of time. Figure 7 shows curves of the proportion of time for
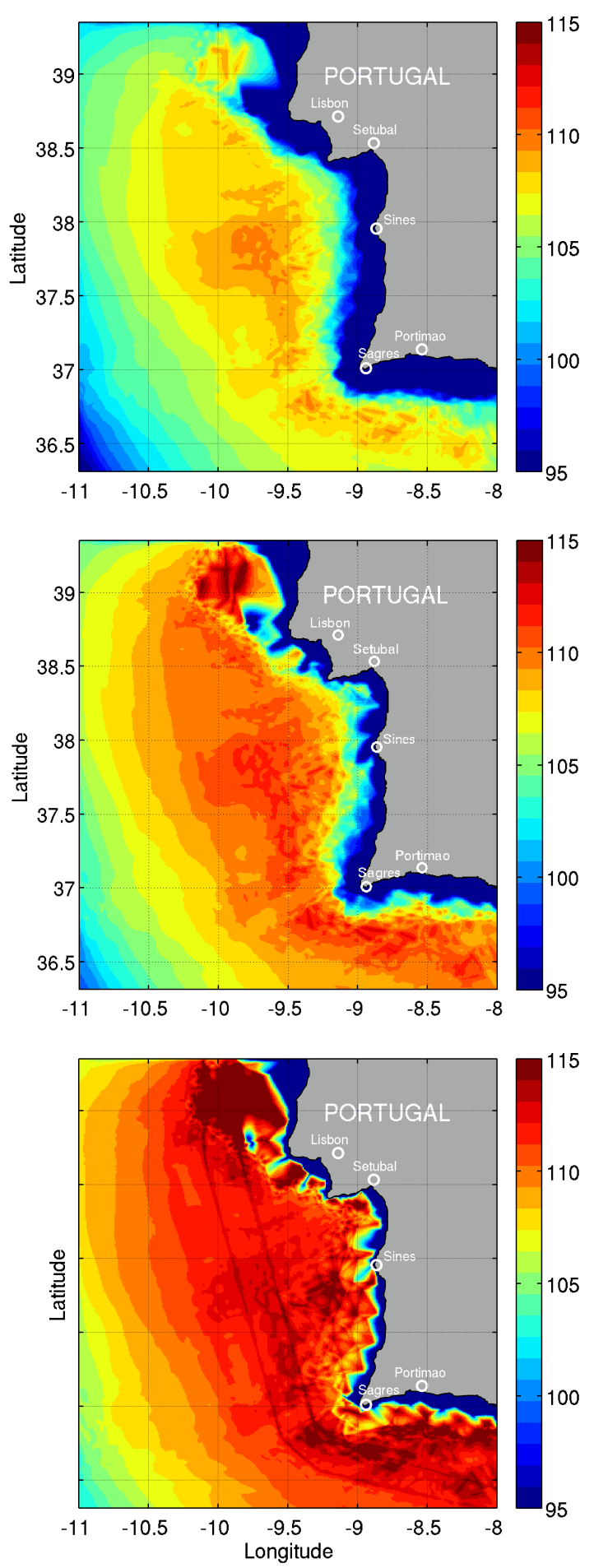

Fig. 6. Predicted SPL as level exceedance [dB $r$ e to $1 \mu \mathrm{Pa}$ ]: exceedence $95 \%$ of the time (top); $50 \%$ of the time (middle); and $5 \%$ of the time (bottom).

which a given level is exceeded for 6 positions arbitrarily selected within the study area, horizontaly aligned over latitude $36^{\circ} 30.00 \mathrm{~N}$ or vertically aligned over longitude $10^{\circ} 00.00 \mathrm{~W}$. The upper/right plot indicates the location for each curve in the longitude and latitude plane. Each plot contains three color coded curves together with an indication, on the lower left 

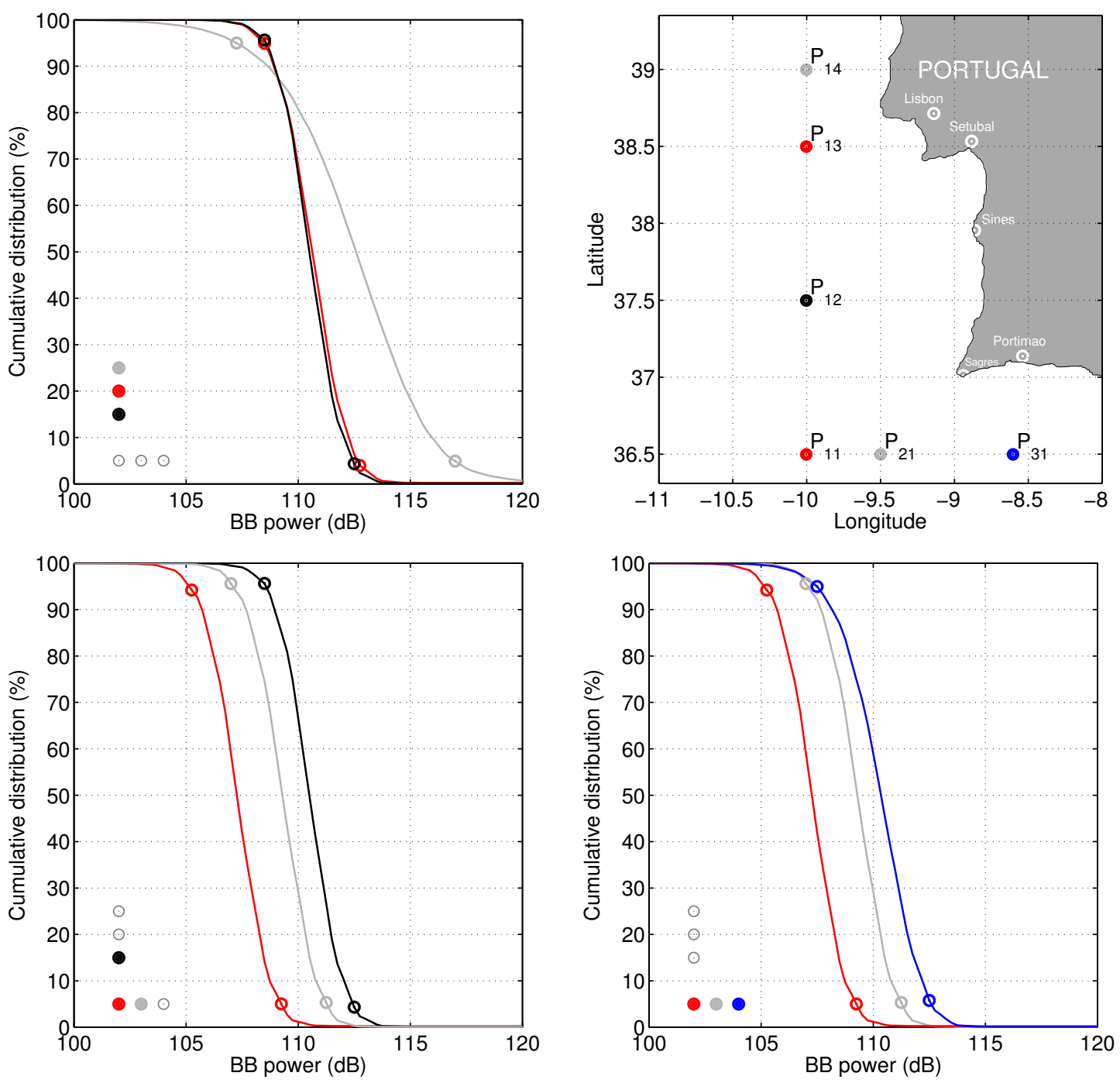

Fig. 7. Predicted SPL as level exceedance in proportion of time for six selected locations over the area: indication of selected locations coded with colors (upper/right); proportion of time where a given minimum level is reached (upper/left and bottom panels).

corner, of the respective geographical positions referred to in the upper right panel figure. The black curve in the upper left plot is repeated in the lower left plot, and the red and gray curves in lower left plot are repeated in lower right plot. This set of plots allow for a comparative evaluation of the exceeded noise level over the study area. Position $\mathrm{P}_{11}$ is that with the lowest level exceedance overall, for being located away from the shipping lanes. Comparatively, positions $\mathrm{P}_{12}$ and $\mathrm{P}_{21}$ are relatively close to the main shipping lanes, with an increase in exceeded levels of approximately 2 and $3.5 \mathrm{~dB}$, respectively. Positions $\mathrm{P}_{12}, \mathrm{P}_{13}$, and $\mathrm{P}_{31}$ all show very similar level exceedance curves, whereas $\mathrm{P}_{12}$ and $\mathrm{P}_{13}$ are located westwards from the shipping lanes, while $\mathrm{P}_{31}$ lies just in the middle of the four lanes. Position $\mathrm{P}_{14}$ is that with the broadest varibility in terms of level exceedance with one of the lowest lower bound and the highest upper bound, overall. This comparatively increased range of variation is attributed to the bathymetry, as $\mathrm{P}_{14}$ is located in a zone with water depth of about $1000 \mathrm{~m}$, while the remaining positions lie in zones with water depth exceeding $2000 \mathrm{~m}$. This case study illustrates the importance of analysing level exceedance in terms of proportion of time in the assessment of noise level at a given position, or for an increased spatial scope.

\section{CONCLUSIONS}

A shipping noise prediction tool has been implemented and used for a case study in Portuguese waters, over a relevant area for carrying very important commercial routes, and for containing several sensitive cases in terms of local fauna, as for example, a resident dolphin population in the Sado River. The prediction tool generates instantaneous noise pictures corresponding to segments of AIS data over 10 minutes intervals, as samples of SPL over the study representing the acoustic time series. This time series is post-processed for noise assessment. Three complementary statistical indicators were used for noise assessment, including average noise levels over the 
longitude/latitude plane; level exceedance for $95 \%, 50 \%$, and $5 \%$ of the over longitude/latitude plane; and level exceedance obtained in terms of cummulative distributions for six selected positions. The statistical indicators provide information on the soundscape over space and time, obtained over a relatively long time series, which inmplies that information on the level of ambient noise over the study area has been generated, in particular, by describing its distribution over space, and its boundaries over significant proportions of time.

This tool may bring significant contributions to the implementation of a regulation such as the European Marine Strategy Framework Directive, which includes underwater noise as a descriptor to be systematically monitored, as it can generate meaningful information to cope with scarce knowledge on the underwater noise in regional areas.

\section{REFERENCES}

[1] M. F. McKenna, D. Ross, S. M. Wiggins, and J. A. Hildebrand. Underwater radiated noise from modern commercial ships. The Journal of the Acoustical Society of America, 131(1):92-103, 2012.

[2] L. Hatch, C. Clark, R. Merrick, S. Parijs, D. Ponirakis, K. Schwehr, M. Thompson, and D. Wiley. Characterizing the relative contributions of large vessels to total ocean noise fields: A case study using the gerry e. studds stellwagen bank national marine sanctuary. Environmental Management, 42:735-752, 2008.

[3] Stephen C. Wales and Richard M. Heitmeyer. An ensemble source spectra model for merchant ship-radiated noise. J. Acoust. Soc. Am., 111(3), 2002.

[4] The European Commission Directive 2008/56/EC of the European Parliament and of the Council of 17 June 2008 establishing a framework for community action in the field of marine environmental policy (Marine Strategy Framework Directive).

[5] A. J. Van der Graaf, M. A. Ainslie, M. André, K. Brensing, J. Dalen, R. P. A. Dekeling, S. Robinson, M. L. Tasker, F. Thomsen, and S. Werner. European marine strategy framework directive - good environmental status (msfd ges): Report of the technical subgroup on underwater noise and other forms of energy. Technical report, 2012.

[6] M. Porter and L. Henderson. Global ocean soundscapes. Proceedings of Meetings on Acoustics, 19(1):-, 2013.

[7] M.E.G.D. Colin, B. Binnerts, M.A. Ainslie, A.M. Benda-Beckmann, C. Booth, N. Bouton, C.M. Harris, C.A.F. de Jong, H.O. Sertlek, and H.W. Slabbekoorn. Ship sound mapping in the north sea. In Proceedings 2nd International Conference on Underwater Acoustics, pages 871-878, 2014.

[8] Michael Porter and Edward L. Reiss. A numerical method for ocean acoustic normal modes. The Journal of the Acoustical Society of America, 76(1), 1984.

[9] W. A. Kuperman, M. B. Porter, J. S. Perkins, and R. B. Evans. Rapid computation of acoustic fields in three-dimensional ocean environments. J. Acoust. Soc. Am., 89(1), 1991.

[10] Richard M. Heitmeyer, Stephen C. Wales, and Lisa A. Pflug. Shipping noise predictions: Capabilities and limitations. Marine Technology Society Journal, 37(4):54-65, 2003-12-01T00:00:00.

[11] Edwin L. Hamilton. Geoacoustic modeling of the sea floor. The Journal of the Acoustical Society of America, 68(5), 1980. 